\title{
Decreased incidence of intussusception during the COVID-19 pandemic. Trends in pediatric surgical emergencies
}

\author{
Shogo Seo ${ }^{1} \cdot$ Kazuto Suda $^{1} \cdot$ Haruki Kato $^{2} \cdot$ Eri Abe $^{3} \cdot$ Seitaro Kosaka $^{1} \cdot$ Kentaro Fujiwara $^{1} \cdot$ Takanori Ochi $^{1}$. \\ Hiroyuki Koga ${ }^{1} \cdot$ Masahiko Urao $^{3} \cdot$ Tadaharu Okazaki $^{2} \cdot$ Geoffrey J. Lane ${ }^{1} \cdot$ Atsuyuki Yamataka $^{1}$
}

Accepted: 1 August 2021 / Published online: 1 September 2021

(c) The Author(s), under exclusive licence to Springer-Verlag GmbH Germany, part of Springer Nature 2021

\begin{abstract}
Purpose Recent reports suggest that the COVID-19 pandemic may be influencing disease morbidity. The purpose of this study was to investigate pandemic-related changes in the incidence of pediatric surgical emergencies.

Methods Data from patients with one of 8 typical conditions considered to be pediatric emergencies who presented at 3 hospitals close to central Tokyo were collated retrospectively from accident and emergency (AE) department records for 2020 and compared with data for 3 years prior to 2020 .

Results All subjects had similar demographic profiles. The total number of pediatric AE attendances from 2017 to 2020 was 2880 (2017: $n=600,2018: n=736,2019: n=817$, and 2020: $n=727$ ). Annual attendances were similar. Of the 8 conditions, there were significantly less cases of intussusception in 2020 than previously $(23 / 727 ; 3.1 \%$ versus $132 / 2153 ; 6.1 \%)$ $p<0.01$ and the number of emergency surgical interventions for intussusception was also significantly less in 2020 (0/23; $0 \%$ versus $13 / 132 ; 9.8 \%) p<0.01$.

Conclusion The implementation of preventative measures to combat the COVID-19 pandemic in 2020 would appear to have influenced the etiopathogenesis of intussusception enough to significantly decrease its overall incidence and the requirement for emergency surgical intervention.
\end{abstract}

Keywords COVID-19 $\cdot$ Intussusception $\cdot$ Pediatric surgical emergency $\cdot$ Pandemic $\cdot$ Emergency department

\section{Introduction}

Severe acute respiratory syndrome coronavirus 2 (SARSCoV-2) and the disease it causes, coronavirus disease 2019 (COVID-19), are causing rapid and tragic health emergencies worldwide $[1,2]$. All aspects of hitherto commonplace customs and daily activities are currently subject to a spectrum of restrictions and health care services are straining to

Shogo Seo

sseo@juntendo.ac.jp

1 Department of Pediatric General and Urogenital Surgery, Juntendo University School of Medicine, 2-1-1 Hongo Bunkyo-ku, Tokyo 113-8421, Japan

2 Department of Pediatric Surgery, Juntendo University Urayasu Hospital, 2-1-1 Tomioka, Urayasu-shi, Chiba 279-0021, Japan

3 Department of Pediatric Surgery, Juntendo University Nerima Hospital, 3-1-10 Takanodai Nerima-ku, Tokyo 177-8521, Japan meet the extra demands of the pandemic while continuing to provide routine care for common health issues.

The COVID-19 pandemic has seen the introduction of universal public health directives designed to control transmission of the virus that have resulted in widespread restrictions on socializing, travel, business, and health care. Continuous use of face masks in public, regular hand washing, use of sanitizing agents, temperature checks, social distancing, closure of shopping malls and teaching institutions, bans on public gatherings, mass testing, quarantine, and lockdowns are now the norm and such control measures have contributed indirectly to prevent trauma [3] and seasonal viral infections $[4,5]$. While there is no doubt that the COVID-19 virus and its variants hinder emergency medicine by complicating procedures because of the need to protect against possible transmission of infection from body fluids and respiratory aerosols, the exact effect of the pandemic on pediatric surgical emergencies is largely unknown because the conditions and circumstances that can cause a child to have a surgical emergency as a result of congenital 
anomalies, trauma, infections, and accidental ingestion/aspiration are so variable.

The purpose of this study was to investigate the effect of the COVID-19 pandemic on the incidence of pediatric surgical emergencies at 3 affiliated centers.

\section{Materials and methods}

To investigate the impact of the COVID-19 pandemic on urgent pediatric surgical practice, we compared accident and emergency (AE) department attendances at 3 affiliated hospitals close to central Tokyo (Juntendo University Hospital, Juntendo University Urayasu Hospital, and Juntendo University Nerima Hospital) by pediatric patients aged 15 or less in 2020 with data from the same centers for 3 years from 2017 to 2019 as controls. All centers involved in this study have AE departments that are readily accessible at all hours and all are accredited as emergency pediatric surgical facilities with comprehensive auxiliary services also available at all hours.

Eight conditions typically considered to be pediatric surgical emergencies (appendicitis, incarcerated inguinal hernia, midgut volvulus, intussusception, acute scrotum, pyloric stenosis, accidental ingestion/aspiration, and trauma) were assessed in this study.

All data were collated retrospectively from AE department databases and individual patient medical records. Statistical analysis was performed using the chi-squared test (Prism software version 8.0) with $p<0.05$ as the limit for significance. Data for two conditions with small sample sizes (midgut volvulus and pyloric stenosis) were analyzed using Fisher's exact test according to Cochran's theorem.

\section{Results}

The total number of all AE department attendances for urgent pediatric surgical consultation at all 3 centers from 2017 to 2020 , was 2880 patients. Annual breakdown was $2017(n=600), 2018(n=736), 2019(n=817)$, and 2020 $(n=727)$. Annual attendances were similar for each year of this 4 year study.

The number of all pediatric AE attendances is summarized per year in Table 1 and per month for 2020 with controls in Fig. 1. Of the 8 emergency pediatric surgical conditions studied, there were no statistically significant differences between 2020 and the 3 years prior to 2020 for the incidences of appendicitis $(127 / 727 ; 17.5 \%$ versus $361 / 2153 ; 16.8 \%)$, incarcerated inguinal hernia $(51 / 727$; $7.0 \%$ versus $185 / 2153 ; 8.6 \%)$, midgut volvulus $(3 / 727$; $0.4 \%$ versus $3 / 2153 ; 0.1 \%)$, acute scrotum $(34 / 727 ; 4.7 \%$ versus $146 / 2153 ; 6.8 \%)$, pyloric stenosis $(8 / 727 ; 1.1 \%$ versus
Table. 1 All accident and emergency department consultations during the study period (2017-2020)

\begin{tabular}{lllll}
\hline & 2017 & 2018 & 2019 & 2020 \\
\hline Appendicitis & 99 & 125 & 137 & 127 \\
Incarcerated inguinal hernia & 61 & 57 & 67 & 51 \\
Midgut volvulus & 1 & 1 & 1 & 3 \\
Intussusception & 39 & 42 & 51 & 23 \\
Acute scrotum & 37 & 54 & 55 & 34 \\
Pyloric stenosis & 9 & 15 & 12 & 8 \\
Accidental ingestion/aspiration & 41 & 57 & 29 & 35 \\
Trauma & 24 & 22 & 33 & 39 \\
Other conditions & 289 & 363 & 432 & 407 \\
Total & 600 & 736 & 817 & 727 \\
\hline
\end{tabular}

$36 / 2153 ; 1.7 \%)$, and accidental ingestion/aspiration (35/727; $4.8 \%$ versus $127 / 2153 ; 5.9 \%$ ).

The incidence of trauma-related urgent pediatric surgical consultations increased in 2020 compared with controls but the difference was not statistically significant $(39 / 727 ; 5.4 \%$ versus $79 / 2153$; 3.7\%) $p=0.06$; Table 1 . Of these, hospitalization was required for $13 / 39(33.3 \%)$ in 2020 and 23/79 $(29.1 \%)$ in controls and emergency surgical intervention was required for $4 / 39(10.3 \%)$ in 2020 and $10 / 79$ (12.7\%) in controls.

Unexpectedly, the number of intussusception cases decreased significantly in 2020 compared with controls $(23 / 727 ; 3.1 \%$ versus $132 / 2153 ; 6.1 \%) p<0.01$; Table 1 , Fig. 2. The requirement for emergency surgical intervention for intussusception also decreased significantly in 2020 from $13 / 132$ cases $(9.8 \%)$ to $0 / 23$ cases $(0 \%) p<0.01$. Two cases of intussusception secondary to malignant tumors (1 case in 2020 and 1 case in controls) were excluded.

\section{Discussion}

In this study, while patients with most of the conditions we investigated continued to present to $\mathrm{AE}$ without change in 2020, there was a decrease in intussusception that was statistically significant and an increase in trauma that was not statistically significant. AE visits appeared to decrease during the lenient form of lockdown called "state of emergency" that was declared around Tokyo on 7 April 2020, extended to cover the whole country on 16 April, and lifted on 25 May 2020, reaching a minimum in June 2020 as non-urgent patients refrained from attending AE for fear of COVID-19.

Intussusception is known to be associated with viral infections, such as respiratory adenovirus [6] and rotavirus [7]. On the other hand, there have been reports of intussusception in an infant as a manifestation of COVID-19 [8, 9], even though pediatric patients seem to have less incidence 


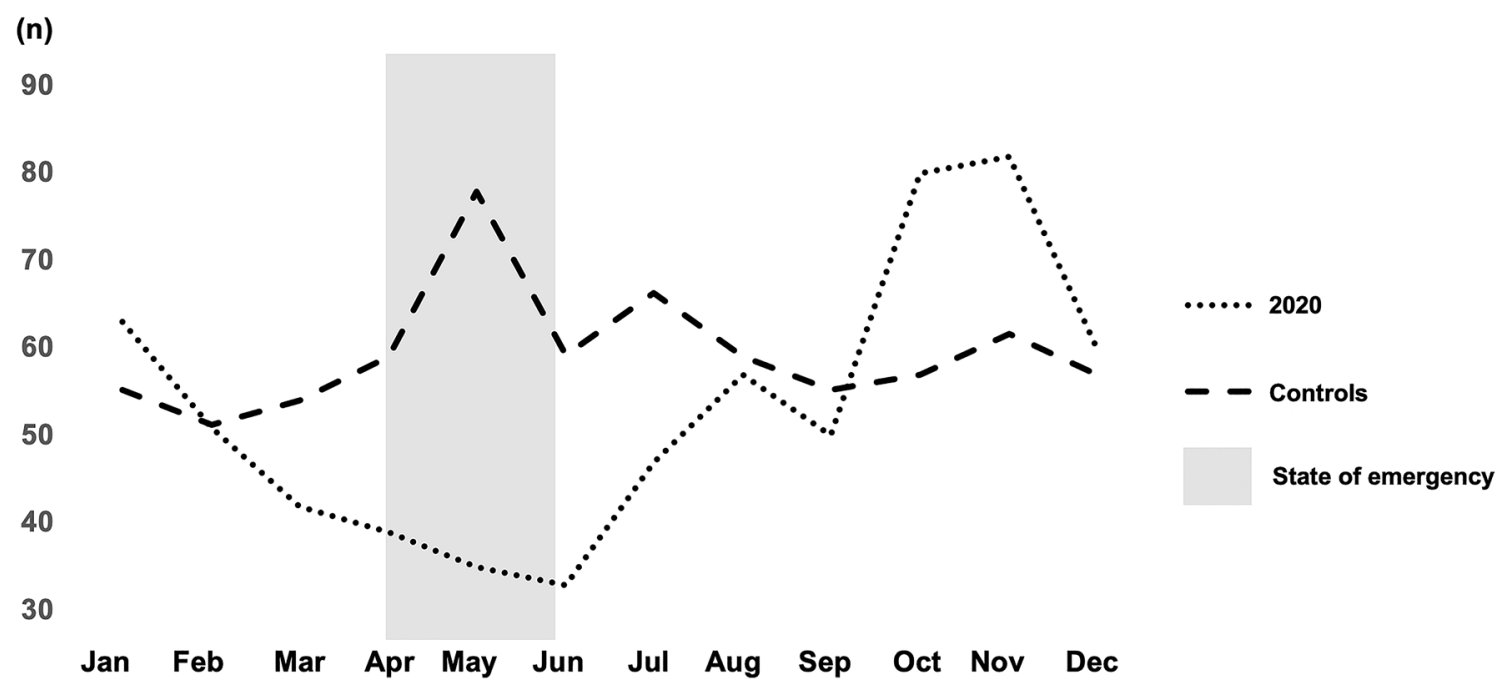

Fig. 1 Monthly accident and emergency department consultations in 2020 versus the average for 3 years (2017-2019)

\section{Incidence of intussusception}

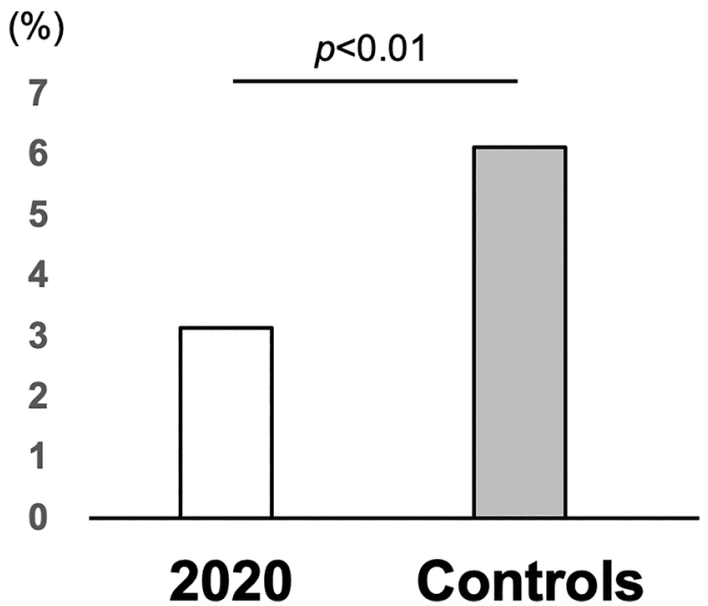

Fig. 2 Proportion of pediatric surgical emergencies caused by intussusception for $2020(23 / 727 ; 3.1 \%)$ versus controls $(132 / 2153 ; 6.1 \%)$

of COVID-19 overall; the mechanism by which this could occur in pediatric patients is not understood and could in fact be idiopathic [10]. With the current pandemic, widespread implementation of measures to prevent transmission of COVID-19, such as hand washing, gargling, mask wearing, and social distancing, has contributed to reducing the incidence of infectious viral diseases $[4,5]$. While we could not identify any scientific reports about the incidence of other infections to support this hypothesis, preventive measures against COVID-19 would appear to be associated with the decrease in intussusception by reducing antecedent viral infections.

Glenn et al. reported a marked reduction in pediatric trauma, $62 \%$ decrease during a period of lockdown compared with an average for the same period over 5 years [3]. Similarly, reduced motor vehicle accidents during lockdowns has been newsworthy internationally $[11,12]$. However, in this study, the incidence of trauma appeared to increase in 2020 although the difference was not statistically significant. The state of emergency declared in Japan was not strictly enforced by any authorities; rather, the public was only advised to limit their activities and recommended to stay home. The increase in traumarelated urgent pediatric surgical consultations observed in 2020 could be influenced by differences in lockdown restrictions enforced in different countries, in other words, Japanese restrictions were not strict enough to decrease trauma as observed in the West. A breakdown of "trauma" would be an interesting topic as a follow-up to this study given the differences in lockdown restrictions enforced internationally and in Japan to understand differences in the incidence of "trauma".

A limitation of this study is that it was based on data collected from 3 affiliated hospitals that are all close to central Tokyo. The urban lifestyle close to central Tokyo is not typical of lifestyles throughout Japan but the difference in incidence between 2020 and controls (excluding the 2 cases of secondary intussusception caused by malignancies) is important enough to suggest that preventive measures designed to combat the transmission of pandemic-related viruses may have indirectly influenced the etiopathogenesis of intussusception. A larger study involving a more representative cross section of hospitals would contribute to understanding the significant decrease in intussusceptions at 3 affiliated hospitals close to central Tokyo in 2020, compared with the 3 years prior to 2020 .

Acknowledgements None 
Author contribution All authors contributed to the study conception and design. Data collection and analysis were performed by SS, KS, HK, EA, and KF. The first draft of the manuscript was written by SS and all authors participated in revising the manuscript. Geoffrey Lane supervised the final English version of the manuscript. All authors read and approved the final manuscript.

Funding No funds, grants, or other support was received.

\section{Declarations}

Conflict of interest The authors declare they have no known competing financial interests or personal relationships that could have influenced this paper.

Ethics approval This study was approved by the Juntendo University School of Medicine Institutional Review Board (No: 20-308) and complies with the Helsinki Declaration of 1975 (revised 1983).

Consent to participate Informed consent was obtained from all individual participants included in the study.

\section{References}

1. Wang D, Hu B, Hu C et al (2020) Clinical characteristics of 138 hospitalized patients with 2019 novel coronavirus-infected pneumonia in Wuhan. China JAMA 323:1061. https://doi.org/10.1001/ jama.2020.1585

2. Remuzzi A, Remuzzi G (2020) COVID-19 and Italy: what next? The Lancet 395:1225-1228. https://doi.org/10.1016/s01406736(20)30627-9

3. Keays G, Friedman D, Gagnon I (2020) Injuries in the time of COVID-19. Health Promot Chronic Dis Prev Can. 40(1112):336-341. Doi. https://doi.org/10.24095/hpcdp.40.11/12.02

4. Lai CC, Chen SY, Yen MY et al (2020) The impact of COVID19 preventative measures on airborne/droplet-transmitted infectious diseases in Taiwan. J Infect S0163-4453(20):30724-30726. https://doi.org/10.1016/j.jinf.2020.11.029
5. Soo RJJ, Chiew CJ, Ma S et al (2020) Decreased influenza incidence under COVID-19 control measures. Singapore Emerg Infect Dis 26(8):1933. https://doi.org/10.3201/eid2608.201229

6. Montgomery EA, Popek EJ (1994) Intussusception, adenovirus, and children: a brief reaffirmation. Hum Pathol 25(2):169-174. https://doi.org/10.1016/0046-8177(94)90274-7

7. Konno T, Suzuki H, Kutsuzawa T et al (1978) Human rotavirus infection in infants and young children with intussusception. J Med Virol 2(3):265-269. https://doi.org/10.1002/jmv.1890020310

8. Giovanni JE, Hrapcak S, Melgar M et al (2021) Global Reports of Intussusception in Infants With SARS-CoV-2 Infection. Pediatr Infect Dis J. 40(1):e35-e36. https://doi.org/10.1097/inf.00000 00000002946

9. Makrinioti H, MacDonald A, Lu X et al (2020) Intussusception in 2 children with severe acute respiratory syndrome coronavirus -2 infection. J Pediatric Infect Dis Soc. 9(4):504-506. https://doi. org/10.1093/jpids/piaa096

10. Athamnah MN, Masade S, Hamdallah H et al (2021) COVID-19 presenting as intussusception in infants: a case report with literature review. J Pediatr Surg Case Rep 66:101779. https://doi.org/ 10.1016/j.epsc.2021.101779

11. Martínez-Castaño I, Calabuig-Barbero E, Gonzálvez-Piñera J et al (2020) COVID-19 infection is a diagnostic challenge in infants with ileocecal intussusception. Pediatr Emerg Care 36(6):e368. https://doi.org/10.1097/pec.0000000000002155

12. Shilling F (2020) Special Report 3: impact of COVID19 mitigation on traffic, fuel use and climate change. Transportation research board. https://roadecology.ucdavis.edu/files/content/ projects/COVID_CHIPs_Impacts_updated_430.pdf [cited 2020 May 28].

13. Kopf D (2020) Traffic collisions are plumme $\neg$ ting in several US cities. Quartz. https://qz.com/1822492/traffic-accidents-areplummeting-because-of-the-pandemic/ [cited 2020 May 28].

Publisher's Note Springer Nature remains neutral with regard to jurisdictional claims in published maps and institutional affiliations. 